BMJ Open Diabetes Research \& Care

\section{Development and validation of the DIabetes Severity SCOre (DISSCO) in 139626 individuals with type 2 diabetes: a retrospective cohort study}

To cite: Zghebi SS, Mamas MA, Ashcroft DM, et al. Development and validation of the Dlabetes Severity SCOre (DISSCO) in 139626 individuals with type 2 diabetes: a retrospective cohort study. BMJ Open Diab Res Care 2020;8:e000962. doi:10.1136/ bmjdrc-2019-000962

- Additional material is published online only. To view please visit the journal online (http://dx.doi.org/10.1136/ bmjdrc-2019-000962).

For 'Presented at statement' see end of article.

Received 8 October 2019 Revised 5 March 2020 Accepted 12 March 2020

\section{Check for updates}

(c) Author(s) (or their employer(s)) 2020. Re-use permitted under CC BY. Published by BMJ.

For numbered affiliations see end of article.

Correspondence to Dr Salwa S Zghebi; salwa.zghebi@manchester. ac.uk

\section{ABSTRACT}

Objective Clinically applicable diabetes severity measures are lacking, with no previous studies comparing their predictive value with glycated hemoglobin $\left(\mathrm{HbA}_{1 \mathrm{c}}\right)$. We developed and validated a type 2 diabetes severity score (the Dlabetes Severity SCOre, DISSCO) and evaluated its association with risks of hospitalization and mortality, assessing its additional risk information to sociodemographic factors and $\mathrm{HbA}_{1 \mathrm{c}}$.

Research design and methods We used UK primary and secondary care data for 139626 individuals with type 2 diabetes between 2007 and 2017, aged $\geq 35$ years, and registered in general practices in England. The study cohort was randomly divided into a training cohort $(\mathrm{n}=111748,80 \%)$ to develop the severity tool and a validation cohort $(n=27878)$. We developed baseline and longitudinal severity scores using 34 diabetes-related domains. Cox regression models (adjusted for age, gender, ethnicity, deprivation, and $\mathrm{HbA}_{1 \mathrm{c}}$ ) were used for primary (all-cause mortality) and secondary (hospitalization due to any cause, diabetes, hypoglycemia, or cardiovascular disease or procedures) outcomes. Likelihood ratio (LR) tests were fitted to assess the significance of adding DISSCO to the sociodemographics and $\mathrm{HbA}_{1 \mathrm{c}}$ models.

Results A total of 139626 patients registered in 400 general practices, aged $63 \pm 12$ years were included, $45 \%$ of whom were women, $83 \%$ were White, and $18 \%$ were from deprived areas. The mean baseline severity score was $1.3 \pm 2.0$. Overall, 27362 (20\%) people died and 99951 (72\%) had $\geq 1$ hospitalization. In the training cohort, a one-unit increase in baseline DISSCO was associated with higher hazard of mortality (HR: $1.14,95 \% \mathrm{Cl}$ 1.13 to 1.15 , area under the receiver operating characteristics curve $(A U R O C)=0.76)$ and cardiovascular hospitalization (HR: $1.45,95 \% \mathrm{Cl} 1.43$ to $1.46, \mathrm{AUROC}=0.73)$. The LR tests showed that adding DISSCO to sociodemographic variables significantly improved the predictive value of survival models, outperforming the added value of $\mathrm{HbA}_{1 \mathrm{c}}$ for all outcomes. Findings were consistent in the validation cohort.

Conclusions Higher levels of DISSCO are associated with higher risks for hospital admissions and mortality. The new severity score had higher predictive value than the proxy used in clinical practice, $\mathrm{HbA}_{1 \mathrm{c}}$. This reproducible algorithm can help practitioners stratify clinical care of patients with type 2 diabetes.

\section{INTRODUCTION}

Diabetes mellitus is a global epidemic with a rapidly increasing prevalence. The WHO

\section{Significance of this study}

What is already known about this subject?

- The prevalence of type 2 diabetes is rapidly increasing worldwide, with associated burdens of morbidity and excess mortality, but validated type 2 diabetes severity measures derived from real-world health records are lacking, as are applications of such measures in clinical practice, despite the importance of assessing diabetes severity being well recognized.

What are the new findings?

- The new Dlabetes Severity SCOre (DISSCO), developed using 34 severity domains coded in routinely collected electronic health records, had overall better predictive value than glycated hemoglobin and showed that people with higher levels of the scores were at up to $45 \%$ significantly increased risk of hospital admission and death.

- DISSCO shows that diabetes-specific severity measures using electronic health records are feasible, with many applications directly relevant to clinical practice and risk stratification in populations.

- The methodology driven by routinely collected medical data is applicable to other chronic conditions managed in primary care setting.

How might these results change the focus of research or clinical practice?

- The results can inform risk stratification for people with type 2 diabetes based on disease severity to support clinicians in providing better self-care and efficient diabetes management in primary care.

- The methodology is applicable to other conditions using routinely collected medical data in future research.

placed diabetes as the seventh main cause of death in 2016. ${ }^{1}$ The cost of diabetes is estimated to be almost one-tenth of the total national healthcare budget in the UK and 
the USA. ${ }^{2}$ Type 2 diabetes affects nearly $90 \%$ of all people with diabetes, ${ }^{4}$ and leads to higher morbidity and mortality related to complications including vascular disease, renal failure, amputations and blindness, in comparison with people without diabetes. ${ }^{56}$

Despite the clinical importance of diabetes and its wider impact on healthcare, summary measures of diabetes severity and their relationship with clinical outcomes have not been widely considered. The 'severity' of a clinical condition is commonly defined via interlinked concepts related to the progression of the underlying processes of the disease. ${ }^{7}$ Increasing disease severity and subsequent development of associated complications lead to greater treatment complexity, healthcare resources utilization and impact on patients' welfare. We sought to develop a new type 2 diabetes severity measure with key focus on disease complications and increased risks for adverse events and death. In current clinical practice, glycated hemoglobin $\left(\mathrm{HbA}_{1 \mathrm{c}}\right)$ is used to summarize glycemic control over the preceding 3 months and might be used as a simple proxy for disease severity, guiding interventions and management. However, $\mathrm{HbA}_{1 c}$ is a unidimensional measure, making it a poor predictor of adverse outcomes. Other generic tools to assess severity of longterm conditions exist, for example the Duke Severity of Illness Checklist, ${ }^{8}$ the Charlson Comorbidity Index, ${ }^{9}$ and the Elixhauser Comorbidity Index. ${ }^{10}$ However, the applicability of these tools to type 2 diabetes is unclear. Logically, a comorbidity-aware disease severity score developed around a driving, index condition may be more predictive than a general measure of comorbidity burden.

Our recent systematic review found that a few studies have assessed or quantified disease severity among individuals with type 2 diabetes and fewer had useful longitudinal measures. ${ }^{11-14}$ We identified the need for a valid and reliable tool for measuring severity of type 2 diabetes and that can serve as an actionable tool for therapeutic targets, as a covariate in epidemiological research, and for stratifying populations in order to inform resource allocation and support commissioning and public health programs for people with diabetes. ${ }^{7}$ Our study aimed to (1) develop a type 2 diabetes severity score (the DIabetes Severity SCOre, DISSCO), (2) evaluate the association and the added clinical utility of the severity score in predicting hospitalization and mortality outcomes beyond that achieved using models incorporating sociodemographic variables and $\mathrm{HbA}_{1 \mathrm{c}}$, and (3) validate DISSCO using a separate cohort (validation data set).

\section{RESEARCH DESIGN AND METHODS}

Data source and patient population

We used the UK Clinical Practice Research Datalink (CPRD) GOLD. CPRD is one of the world's largest electronic health record (EHR) databases providing detailed anonymized medical data and is representative of the UK population. ${ }^{1516} \mathrm{CPRD}$ provides data linkage to additional clinical data sets and disease registries. ${ }^{17} 18$ We used three data linkages: Hospital Episode Statistics (HES), Office for National Statistics (ONS) mortality data, and patientlevel Index of Multiple Deprivation (IMD) quintiles 2015. The planned study design has been reported previously. ${ }^{7}$

A prevalent cohort of patients registered in general practices eligible for data linkage with at least one diagnostic code for type 2 diabetes from 1 March 2007 to 31 March 2017 and aged $\geq 35$ years at diagnosis were identified. Patients who also had a code for type 1 diabetes in their entire data record or an indiscriminate gender were excluded. For each patient, index date was defined as the earliest diabetes diagnosis date. Each patient was followed up from the index date $\left(\mathrm{t}_{0}\right)$ contributing to the survival time in years (t) until the earliest date of developing an outcome of interest, leaving the general practice, study end date (31 March 2017), or death. The final cohort of eligible patients was randomly split at patient level into training ( $80 \%$ of the cohort) and validation (20\% of the cohort) data sets.

\section{Aim 1: diabetes severity assessment Severity domains}

Key clinically relevant indicators of type 2 diabetes severity (severity domains) were identified from a systematic review of studies that quantified type 2 diabetes severity. ${ }^{14}$ We also sought expert clinical opinion in cardiology, diabetes and primary care, supplementing the list of severity domains. $\mathrm{HbA}_{1 c}$ and demographic variables were excluded from the severity domains. We identified 34 severity domains (online supplementary table S1).

\section{Calculation of severity scores}

Using the defined severity domains, we calculated DISSCO using three different methods to assess the baseline and longitudinal levels of type 2 diabetes severity. The methods differed in the weights assigned to each diabetes severity domain when computing the overall severity score. The first and second methods were based on the binary and hierarchical classifications reported previously. ${ }^{7}$

The first method was a simple count $(\mathrm{C})$ of the total number of severity domains present for an individual, out of 29 , by assigning equal weighting (weight of 1 ) to all domains in the overall score (online supplementary table $\mathrm{S} 1$ ). In this method, 29 of the 34 domains were used by merging 8 domains into 3 domains.

The second method produced severity-weighted (SW) scores by assigning a weight to each of the 34 domains according to a hierarchy of increased severity based on clinical judgment as reported previously. ${ }^{7}$ As provided in online supplementary table S2, for example, a hierarchical weight of 1 was assigned to a transient ischemic attack (TIA) event, 2 to an event of carotid artery interventions, and 3 to a record of stroke to indicate the increasing severity of cerebrovascular events. For each patient, the overall SW score was the sum of the total domains' hierarchical weights. 
For the simple count and SW scores, we investigated the effects of varying the length of the pre-index (before diabetes diagnosis index) look-back window, defining the time period within which events had to occur for inclusion in the severity score calculation. We examined three lengths of look-back windows: unlimited (events at any time in the patient's entire pre-index record), up to 10 years before index date, and up to 5 years before index date.

The third method was an exploratory analysis based on weighing the severity domains recorded before index date by proximity to index date, to generate "proximityweighted' (PW) score. This approach used a 5-year preindex window, with any events prior to this window not being counted in the score (ie, weight of 0 ). Events within a year before the index year were assigned a weight of 1 , 2 years a weight of $0.8,3$ years a weight of $0.6,4$ years a weight of 0.4 , and 5 years a weight of 0.2 .

Finally, to account for longitudinal severity that may have developed after the diagnosis of type 2 diabetes (post-index), we explored models incorporating severity domains recorded after the index date. Thus, we calculated 'moving post-index severity scores', allowing the window within which events could occur to range from 1 to 5 years after the index date, each coupled with the three look-back windows (online supplementary figure S1). For example, the 3-year post-index window coupled with the 10-year look-back window ranged from 7 years prior to the index year to 3 years afterwards.

In summary, we calculated 37 severity scores per patient as follows:

- Seven pre-index scores for computing overall severity using the three aforementioned methods:

- A simple count (C) of domains score using the following windows:

1. Unlimited look-back window (CU).

2. 10-year look-back window (C10).

3. 5-year look-back window (C5).

- Severity-weighted (SW) score using:

4. Unlimited look-back window (SWU).

5. 10-year look-back window (SW10).

6. 5-year look-back window (SW5).

- Proximity-weighted (PW) score using the following:

7. 5-year look-back window (PW).

- 30 post-index scores, based on combining each of windows 1-6 with post-index windows length of 1, 2, 3,4 , and 5 years.

\section{Aim 2: evaluating the added predictive value and clinical utility of the severity score}

We evaluated the effect of adding the severity score by comparing with survival models that contain only age, gender, ethnicity, and IMD quintiles. We also investigated whether the inclusion of $\mathrm{HbA}_{1 \mathrm{c}}$ improved the prediction of outcomes by adjusting for baseline $\mathrm{HbA}_{1 \mathrm{c}}$ levels.

\section{Outcomes}

The primary outcome was all-cause mortality. The secondary outcomes were future hospitalizations: any cause, due to cardiovascular (CV), diabetes (hypoglycemia-related hospitalizations; aggregated diabetic microvascular complications, foot ulcers, amputation, gangrene, hyperosmolar hyperglycemic state, and diabetic ketoacidosis), clustered $\mathrm{CV}$ and diabetes, or $\mathrm{CV}$ procedures (as coded by the OPCS Classification of Interventions and Procedures (OPCS-4). Cause-specific hospitalization was identified using the tenth revision International Classification of Diseases (ICD-10) code recorded in the first hospital admission after the index date. The hospitalization and death outcomes were identified using linked hospitalization admitted patient care (HES APC) data and the ONS mortality data, respectively.

\section{Statistical analysis}

Patient characteristics are described by the data set purpose (training and validation cohorts) and by levels of baseline 10-year DISSCO $(0,1,2,3,4,5$, and $\geq 6)$ and presented as mean \pm SD or count $(\%)$. Descriptive statistics of the developed severity scores are reported at baseline (index date) and over time (up to 5 years after index). Social deprivation levels were modeled as IMD quintiles (quintile 1 indicating the least deprived level and quintile 5 as the most deprived level). Patients with missing deprivation level were assigned to a sixth category, 'Unknown'.

Baseline $\mathrm{HbA}_{1 \mathrm{c}}$ was based on the nearest test result recorded within 1 year before and 6 months after the index date. We found $\mathrm{HbA}_{1 \mathrm{c}}$ recording improved over time as the number of patients with $\mathrm{HbA}_{1 \mathrm{c}}$ recorded at baseline increased from $82 \%$ at baseline to $90 \%$ at the study end in both cohorts (online supplementary table S3). If there was no $\mathrm{HbA}_{1 \mathrm{c}}$ recorded during 1 year before to 6 months after index date, then baseline $\mathrm{HbA}_{1 \mathrm{c}}$ was deemed missing for that patient.

The relationships between the sociodemographic and clinical covariates, with and without the new severity score (simple count (C), SW, or PW), and the outcomes were evaluated using four Cox proportional hazards models, reporting HR (95\% CI):

- Model 1: age, gender, deprivation, and ethnicity.

- Model 2: model 1 and severity score.

- Model 3: model 1 and $\mathrm{HbA}_{1 c}$.

- Model 4: model 1, $\mathrm{HbA}_{1 \mathrm{c}}$ and severity score.

The PW score and moving post-index scores were only modeled with the primary outcome (all-cause mortality). Online supplementary tables S4 and S5 summarize the fitted Cox regression models. The level of deprivation was modeled using the least deprived as the referent group and for ethnicity variable, White was the referent group. Baseline $\mathrm{HbA}_{1 \mathrm{c}}$ was modeled using the International Federation of Clinical Chemistry (IFCC) unit of measure $(\mathrm{mmol} / \mathrm{mol})$ divided by 10 for a meaningful interpretation of the results on a 10-unit increase of $\mathrm{HbA}_{1 \mathrm{c}}$.

The Cox models included a severity score to evaluate the association with future outcomes' prediction window (follow-up), that is, from the index date until censoring. The predictive value of all survival models was assessed using Gönen and Heller's K concordance 
statistic (C-statistic), a measure of the area under the receiver operating characteristics (AUROC) curve for censored data. C-statistic ranges between 0 and 1 , where a value of 0.5 indicates no predictive discrimination and a value close to 1 indicates an accurate model with high separation of subjects with different outcomes. ${ }^{19}$ Likelihood ratio (LR) tests were fitted to assess the statistical significance of adding DISSCO to the demographics and $\mathrm{HbA}_{1 \mathrm{c}}$ models in improving the models' fit. The severity scores' calibration was tested using three methods: Somer's D; comparing the survival curves for a given risk group in the training and validation data sets; and predicting population-averaged survival probabilities and comparing the observed and predicted survival probabilities in several prognostic groups derived by the severity score's cut points. The proportional hazards assumption was assessed using Schoenfeld residuals. Data were analyzed using Stata V.15. ${ }^{20}$ The study is reported in accordance with the RECORD (REporting of studies Conducted using Observational Routinely collected health Data) statement. ${ }^{21}$

\section{Aim 3: validation data set}

All analyses were replicated in the validation data set.

\section{Patient and public involvement and engagement (PPIE)}

People with type 2 diabetes were invited to a patient and public involvement and engagement event to seek their views on the included severity domains and the score calculation methods. They were also asked about the readability of a lay summary of the study and for advice about dissemination approaches for reporting the study results. The participants commented on the importance and the relevance of the study and the need to raise awareness on diabetes severity and the involvement of several body organs. Perceptions of severity domains varied among participants, where some participants did not identify that some conditions were related to their diabetes and its progression (such as declined renal function and foot problems), while other participants listed additional indicators such as rapid onset of tiredness and stress. The participants were more interested in the SW score approach and commented that it could include more intermediate domains, such as more stages between laser therapy and blindness, which we explained were often lacking in electronic data. The participants advised to disseminate the study results to patients and clinical audiences including general practices, diabetes centers, and lay audiences using social media.

\section{RESULTS}

\section{Patient population}

A total of 139626 eligible patients with type 2 diabetes were were included in the analysis. The training data set included 111748 patients and the validation data set 27 878 , with a mean $( \pm \mathrm{SD})$ follow-up of $7.6( \pm 4.8)$ years. The mean age of the training and validation cohorts was 63.0 $( \pm 12.5)$ years and $63.1( \pm 12.6)$ years, respectively, of which
$49686(45 \%)$ and $12482(45 \%)$ were women, respectively (table 1). Nearly $12.5 \%$ of people were censored due to transfer out of the general practice during the study (of whom $73 \%$ left the practice due to unknown reasons and $14 \%$ due to death).

Compared with individuals with 10 -year pre-index simple count score (C10) of 0 (no severity domains at baseline), individuals with a score of $\geq 6$ were older $(72.0 \pm 9.5$ vs $57.8 \pm 12.3)$, more likely to be male $(67 \%$ vs $57 \%$ ), and overall similar proportions were living in more deprived areas (21\% vs $18 \%$ ) (online supplementary table S6). CV domains were highly prevalent in those with severity score $\geq 6$.

\section{Severity score (aim 1)}

In the training data set, the simple count and SW scores differed in terms of mean and range by the time of lookback window, and increased over time. The pre-index 10-year count score (C10) ranged between 0 and 12 (online supplementary figure S2A), while the corresponding SW score (SW10) score ranged between 0 and 22 (online supplementary figure S2B and online supplementary table S7). The severity scores calculated using the 10-year look-back windows were very similar to that calculated using unlimited look-back windows. The 10-year lookback captured around $80 \%$ of all domains contributing to severity relative to the unlimited window. The PW score ranged between 0 and 7.6. The distribution of simple count and SW scores at pre-index (online supplementary figure S2) and post-index (online supplementary figures S3 and S4) windows showed similar trend overall.

\section{Survival analyses (aim 2)}

Cox regression models were fitted to assess the relationship between severity score level and all-cause mortality and six future any cause and cause-specific hospitalizations events described. The estimated HRs for severity scores derived by the three different methods are not directly comparable, being based on different scales; therefore, AUROCs were used for comparison across models. Testing for proportional hazards indicated the assumptions held true as shown by selected figures representing each outcome presented in online supplementary figures S5 and S6.

\section{Mortality outcome}

In the training data set, a total of 21969 (20\% of patients) deaths occurred over 848742 patient-years of follow-up. The results show that diabetes severity was positively associated with increasing risk for all-cause mortality (figure 1A). An increase of one-unit in the 10-year simple count (C10) score at the index year was associated with up to $14 \%$ (95\% CI $13 \%$ to $15 \%$, AUROC $=0.76$ ) higher risk for mortality when adjusted for demographics and $\mathrm{HbA}_{1 \mathrm{c}}$ (model 4; online supplementary table S8). Older patients and men were at a greater risk.

Simple count and SW scores measured over moving $1-5$ years post-index windows (moving post-index scores) 
Table 1 Baseline characteristics of the identified study cohort of type 2 diabetes, by full dataset and by training and validation cohortsdatasets

\begin{tabular}{|c|c|c|c|}
\hline Characteristics & Full dataset & Training dataset & Validation datase \\
\hline Patient count, n (\%) & 139626 & $111748(80)$ & $27878(20)$ \\
\hline Age, years $\pm S D$ & $63.0 \pm 12.5$ & $63.0 \pm 12.5$ & $63.1 \pm 12.6$ \\
\hline Gender (female), n (\%) & $62168(44.5)$ & $49686(44.5)$ & $12482(44.8)$ \\
\hline Number of general practices & 400 & 400 & 396 \\
\hline Mean baseline $\mathrm{HbA}_{1 \mathrm{c}}, \%(\mathrm{mmol} / \mathrm{mol} \pm \mathrm{SD})^{*}$ & $7.8(62 \pm 22)$ & $7.8(62 \pm 22)$ & $7.8(62 \pm 22)$ \\
\hline Cases with $\mathrm{HbA}_{1 \mathrm{c}}$ data, $\mathrm{n}(\%)$ & $122294(88)$ & $97858(88)$ & $24436(88)$ \\
\hline \multicolumn{4}{|l|}{ Ethnicity, n (\%) } \\
\hline White & $116393(83.4)$ & $93157(83.4)$ & 23236 (83.3) \\
\hline Non-White & $11024(7.9)$ & $8842(7.9)$ & $2182(7.8)$ \\
\hline Unknown & $12209(8.7)$ & $9749(8.7)$ & $2460(8.8)$ \\
\hline \multicolumn{4}{|l|}{ IMD quintiles, $\mathrm{n}(\%)$} \\
\hline Quintile 1 (affluent) & 26930 (19.3) & 21437 (19.2) & $5493(19.7)$ \\
\hline Quintile 2 & $29534(21.1)$ & $23580(21.1)$ & $5954(21.4)$ \\
\hline Quintile 3 & $29539(21.1)$ & $23742(21.2)$ & $5797(20.8)$ \\
\hline Quintile 4 & $27883(20.0)$ & $22341(20.0)$ & $5542(19.9)$ \\
\hline Quintile 5 (deprived) & 25641 (18.4) & $20571(18.4)$ & $5070(18.2)$ \\
\hline Unknown & $99(0.1)$ & $77(0.1)$ & $22(0.1)$ \\
\hline Mean follow-up, years $\pm S D$ & $7.6 \pm 4.8$ & $7.6 \pm 4.7$ & $7.6 \pm 4.8$ \\
\hline \multicolumn{4}{|l|}{ Region in England, $\mathrm{n}(\%)$} \\
\hline North East & $3202(2.3)$ & $2560(2.3)$ & $642(2.3)$ \\
\hline North West & 24115 (17.3) & $19412(17.4)$ & $4703(16.9)$ \\
\hline Yorkshire and the Humber & $6036(4.3)$ & $4799(4.3)$ & $1237(4.4)$ \\
\hline East Midlands & $4553(3.3)$ & 3660 (3.3) & $893(3.2)$ \\
\hline West Midlands & $17838(12.8)$ & $14252(12.8)$ & $3586(12.9)$ \\
\hline East of England & $14540(10.4)$ & $11573(10.4)$ & 2967 (10.6) \\
\hline South West & $18658(13.4)$ & 14888 (13.3) & $3770(13.5)$ \\
\hline South Central & $15617(11.2)$ & $12441(11.1)$ & $3176(11.4)$ \\
\hline London & $17250(12.4)$ & $13829(12.4)$ & $3421(12.3)$ \\
\hline South East Coast & 17817 (12.8) & 14334 (12.8) & 3483 (12.5) \\
\hline
\end{tabular}

*Most recent measure within 1 year before or 6 months after index date.

$\mathrm{HbA}_{1 \mathrm{c}}$, glycated hemoglobin; IMD, Index of Multiple Deprivation.

showed consistent direction of association as observed with corresponding pre-index scores, indicating the association between a one-unit increase in post-index severity and risk of death (online supplementary tables S9 and S10). The strength of association remained statistically significant at 5 years after index date and a look-back window of up to 10 years (C10) (adjusted HR: 1.16, 95\% CI 1.15 to 1.17 , AUROC=0.75). Similar overall trends were observed with post-index SW scores. Similar estimate was obtained using the PW score in the fully adjusted model (model 4) (HR: 1.18, 95\% CI 1.16 to 1.20 , AUROC=0.75).

\section{Hospitalization outcomes}

Over the follow-up period, a total of 79974 (72\% of patients) hospitalization events of any cause occurred over 409406 patient-years of follow-up (training data set). These included 37134 (33\%) CV-related hospitalizations, 69120 (62\%) diabetes-related hospitalizations, 3268 (3\%) hypoglycemia hospitalizations, 71505 (64\%) clustered $\mathrm{CV}$ and diabetes-related hospitalizations, and 5593 (5\%) CV procedures. Increasing diabetes severity at index year showed a greater risk for future hospitalizations (figure 1B). A one-unit increase in C10 score was associated with $9 \%(95 \%$ CI $8 \%$ to $9 \%$, AUROC $=0.63)$ significantly higher risk for hospital admission of any cause (online supplementary table S11). Severity remained strongly associated with hospitalization outcomes after further adjusting for $\mathrm{HbA}_{1 \mathrm{c}}$ levels.

In the fully adjusted model (model 4), a one-unit higher 10-year simple count score was associated with a $45 \%$ (95\% CI $43 \%$ to $46 \%$ ) greater risk of being 

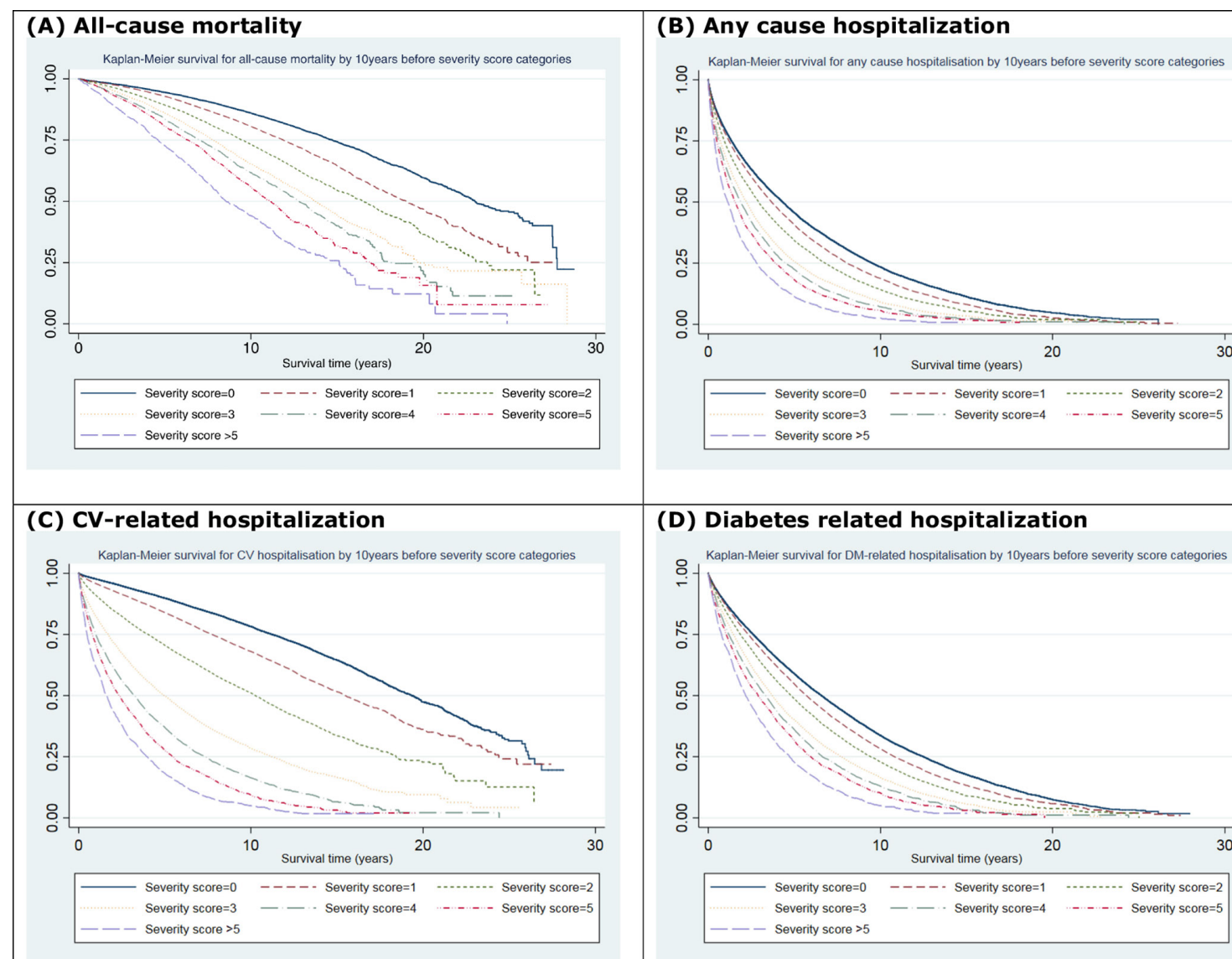

\section{(D) Diabetes related hospitalization}

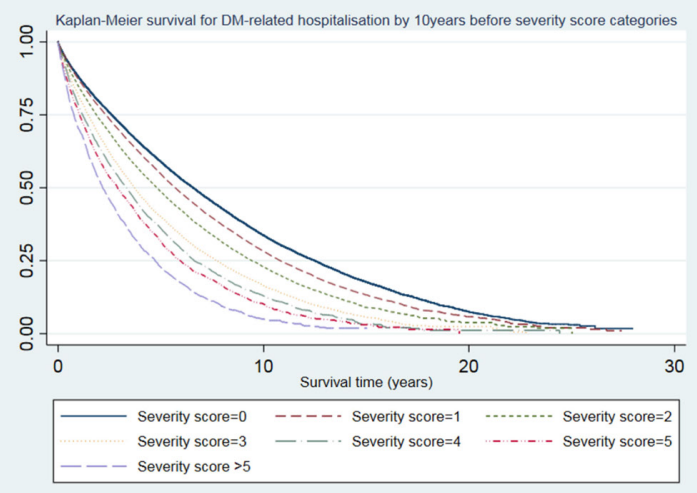

\section{(E) Hypoglycemia-related hospitalization}

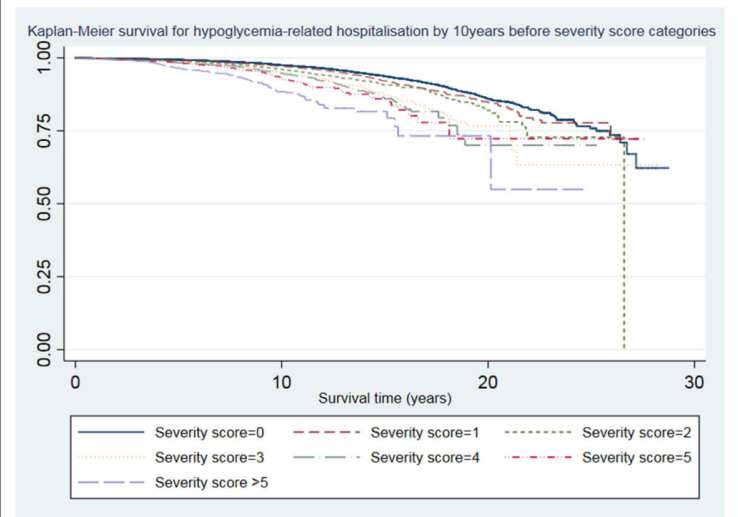

\section{(F) Clustered CV-diabetes relatedhospitalization}

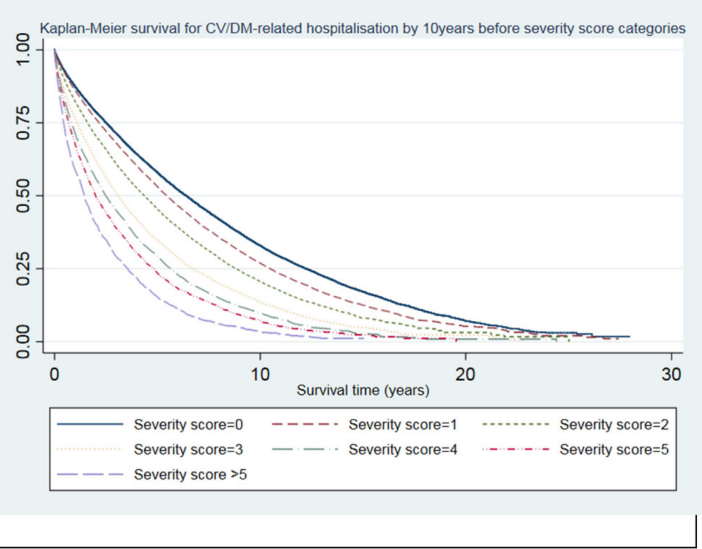

Figure 1 Kaplan-Meier plots for risk of adverse outcomes associated with 10-year (C10) severity score categories: (A) all-cause mortality; (B) any-cause hospitalization; (C) cardiovascular (CV)-related hospitalization; (D) diabetes-related hospitalization; (E) hypoglycemia-related hospitalization; and (F) clustered CV-diabetes related hospitalization-training data set.

hospitalized for cardiovascular disease (AUROC $=0.73$ ) (online supplementary table S12 and figure 1C). The corresponding SW10 score was associated with a $24 \%$ risk (95\% CI $23 \%$ to $24 \%$, AUROC $=0.72$ ).

Individuals with a one-unit higher C10 score were also at greater risk for other cause-specific hospitalization admissions (figure 1D-F): diabetes-related hospitalizations (adjusted HR: $1.10,95 \%$ CI 1.09 to 1.10 , AUROC=0.63) (online supplementary table S13), hypoglycemia-related hospitalizations (adjusted HR: 1.15, 95\% CI 1.12 to 1.18 , AUROC $=0.72$ ) (online supplementary table S14), clustered $\mathrm{CV} /$ diabetes-related events (adjusted HR: 1.14, $95 \%$ CI 1.13 to 1.14 , AUROC=0.64) (online supplementary table S15), and CV interventions (adjusted HR: 1.33, 95\% CI 1.31 to 1.35 , AUROC=0.69) (online supplementary table S16).

Assessing the predictive value of the developed severity score (DISSCO) showed its additive and improved 
predictive value. The AUROC for models with and without severity score shows that adding DISSCO improved the model discrimination for all measured outcomes, as observed in model 2 versus model 1 and in model 4 versus model 3 (table 2 and online supplementary figure S7 and S8). For CV hospitalization, model 3 had an AUROC of 0.71, which increased to an AUROC of 0.73 when C10 score was added (model 4 ), while modest improvements were observed for all-cause mortality and diabetes-related hospitalizations, but all were significant $(p<0.001)$ as shown by LR tests. The performed calibration tests showed good calibration of the severity scores (online supplementary table S17; figure 1 vs online supplementary figures S9, and S10).

\section{Validation data set (aim 3)}

The validation data set included 27878 patients (table 1). The regression analyses conducted in the validation data set (online supplementary tables S18-S22, figure S5) resulted in consistent findings with those reported with the training data set. A summary of the findings in the training and validation data sets is presented in table 3 .

\section{DISCUSSION}

\section{Main findings}

We present a contemporary scoring system using EHRs to grade type 2 diabetes severity in a large primary care cohort. Our findings prove the concept that longitudinal EHRs and linked administrative data can be used to develop type 2 diabetes severity score that is useful for predicting key outcomes, with a methodology applicable to other chronic conditions. The developed baseline and longitudinal severity scores (DISSCO) provided important prognostic information for hospitalization and mortality events. DISSCO improved the predictive value of models for all measured outcomes when added to basic sociodemographic variables and also performed better than adding $\mathrm{HbA}_{1 \mathrm{c}}$ levels. This indicates the value of the included severity domains by mapping to the measured outcomes. The predictive value of pre-index and post-index simple count scores was slightly higher than the SW score. Results were very similar for the 10 -year and unlimited window models. In actual practice systems, patient records can stretch back many decades. We therefore recommend using a window of 10 years to reduce confounding with varying record lengths and less reliable data.

\section{Comparison with other studies}

Our recent systematic review showed some development of diabetes-specific severity scores, mainly using EHRs, in several countries but not in the UK. ${ }^{14}$ Prior studies used either continuous or categorical grading systems based on diabetes-related complications and glycemic indicators, mainly $\mathrm{HbA}_{1}$. In one study, the severity of type 2 diabetes in 300 individuals was categorized into four levels using an automated algorithm based on two domains: insulin use and the presence of diabetes complications. ${ }^{12}$ Another US study assessed severity using two methods involving a number of severity indicators, including diabetes complications and laboratory data. The latter study reported greater risks of hospitalization and death (over a total of 14166 patients-years) with increased type 2 diabetes severity, consistent with our findings. ${ }^{13}$

Despite the knowledge added by these previous models, some studies were limited by design (such as small sample sizes ranging from 65 to 4229 ) or the type of included severity indicators. Importantly, none of the studies compared the severity measures with $\mathrm{HbA}_{1 \mathrm{c}}$, and only a few examined the prospective application of the severity measure to serve as an actionable clinical tool. ${ }^{22} 23$ In comparison, we included a much larger type 2 diabetes cohort $(\mathrm{n}=139$ 626) over a longer follow-up (up to 848742 patient-years) using multiple approaches to assess severity and investigated its association with cause-specific hospitalization. Importantly, table 2 shows the superiority of the added predictive value of DISSCO (model 2) over $\mathrm{HbA}_{1 c}$ (model 3) when added to model 1.

\section{Implications and clinical relevance}

Assessing disease severity for a highly prevalent chronic condition such as type 2 diabetes can have clinical validity beyond that achieved using more traditional approaches (demographic and clinical variables). Well-defined disease severity measurement based on readily collected data has important implications on targeted patient care, utilization of healthcare services and service planning. The focus of this study was on type 2 diabetes; however, future studies are needed to assess the severity of type 1 diabetes, but separately from other forms due to its distinctive phenotype, and epidemiological and pathophysiological differences compared with type 2 diabetes. Our study adds to current knowledge by offering a contemporary and validated type 2 diabetes severity measure driven by data collected in primary care. Given that diabetes is mainly managed in primary care settings, ${ }^{24-28}$ a primary care-derived measure has broad applicability especially when it predicts key outcomes well, as DISSCO does. The proposed algorithm can be initially introduced as a paper-based version (online supplementary table S23) to enable clinicians to calculate baseline and longitudinal severity scores and discuss them with the patient, that is, as a risk stratification tool to identify patients with type 2 diabetes at higher risk for adverse outcomes, which informs advanced decisions with patients and their carers. Future implementation studies are needed to develop DISSCO into an accessible software that can be incorporated in EHRs, such as QRISK and FRAX scores, ${ }^{29}{ }^{30}$ as a practical actionable tool to stratify patients with diabetes by severity of their condition for better self-care and efficient diabetes management in primary care. Additionally, our approach is applicable to most long-term conditions. While acknowledging the different coding systems and other operational/technical differences, some 


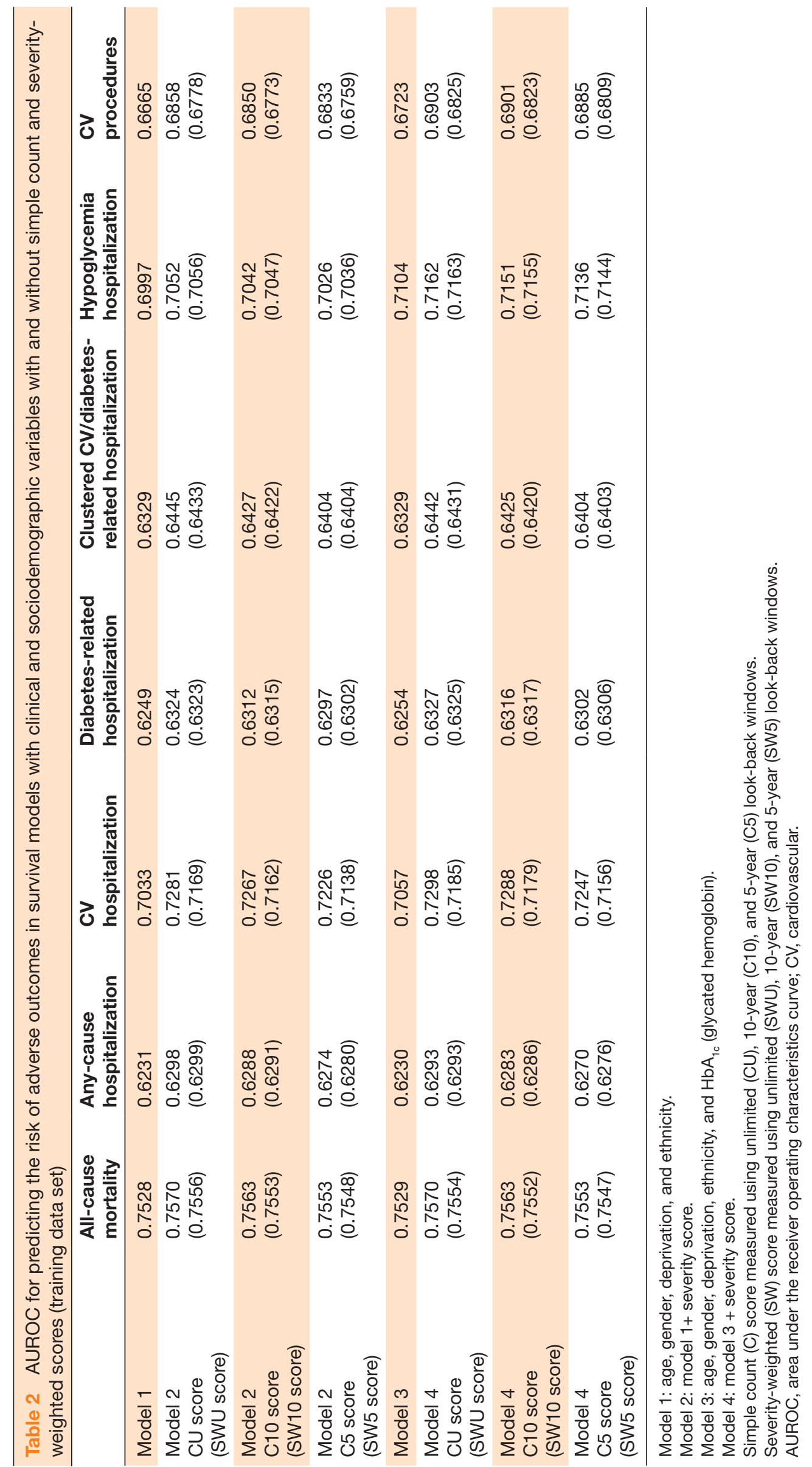

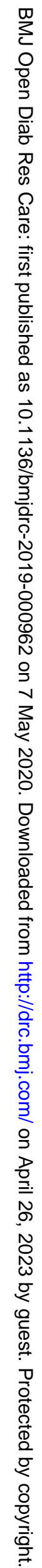


Table $3 \mathrm{HR}(95 \% \mathrm{Cl})$ for risk of primary and secondary outcomes associated with baseline severity score using training and validation data sets

\begin{tabular}{|c|c|c|}
\hline & $\begin{array}{l}\text { Training data set } \\
\text { HR }(95 \% \mathrm{Cl})\end{array}$ & $\begin{array}{l}\text { Validation data set } \\
\text { HR }(95 \% \mathrm{Cl})\end{array}$ \\
\hline \multirow[t]{2}{*}{ All-cause mortality } & 1.14 (1.13 to 1.15$)$ & $1.16(1.14$ to 1.18$)$ \\
\hline & AUROC $=0.7563$ & AUROC $=0.7617$ \\
\hline \multirow[t]{2}{*}{ All-cause hospitalization } & 1.09 (1.08 to 1.09$)$ & 1.09 (1.08 to 1.11$)$ \\
\hline & AUROC $=0.6283$ & AUROC $=0.6320$ \\
\hline \multirow[t]{2}{*}{ CV-related hospitalization } & 1.45 (1.43 to 1.46$)$ & $1.44(1.42$ to 1.46$)$ \\
\hline & AUROC $=0.7288$ & AUROC $=0.7326$ \\
\hline \multirow[t]{2}{*}{ Diabetes-related hospitalization } & 1.10 (1.09 to 1.10$)$ & $1.10(1.08$ to 1.11$)$ \\
\hline & AUROC $=0.6316$ & AUROC $=0.6311$ \\
\hline \multirow[t]{2}{*}{ Clustered CV-related or diabetes-related hospitalization } & 1.14 (1.13 to 1.14$)$ & $1.14(1.13$ to 1.15$)$ \\
\hline & AUROC $=0.6425$ & AUROC=0.6439 \\
\hline \multirow[t]{2}{*}{ Hypoglycemia-related hospitalization } & 1.15 (1.12 to 1.18$)$ & $1.18(1.12$ to 1.24$)$ \\
\hline & AUROC $=0.7151$ & AUROC $=0.7225$ \\
\hline
\end{tabular}

Data based on model 4 (adjusted for baseline $\mathrm{C} 10$ score, age at index, gender, $\mathrm{HbA}_{1 \mathrm{c}}$, deprivation, and ethnicity) (restricted to individuals with non-missing baseline $\mathrm{HbA}_{1 c}$; training data set $\mathrm{n}=97858$ and validation data set $\mathrm{n}=25099$ ).

AUROC, area under the receiver operating characteristics curve; $\mathrm{CV}$, cardiovascular; $\mathrm{HbA}_{1 \mathrm{c}}$, glycated hemoglobin.

international primary care data sets and diabetes registries, such as the Swedish National Diabetes Register, ${ }^{31}$ the Canadian Primary Care Sentinel Surveillance Network, ${ }^{32}$ and the Diabetes Collaborative Registry, ${ }^{33}$ include the majority of our severity domains, indicating the score's reproducibility and potential generalizability in non-UK health systems.

\section{Strengths and limitations of the study}

Our study has several strengths. First, to the best of our knowledge, it is the first large study to measure the severity of diabetes using UK (England) data. Second, we used high-quality clinical primary and secondary care data, and we developed a contemporary severity measure using data collected in routine general practice visits to indicate the potential of our measure as a practical tool in primary care, with a methodology applicable to type 2 diabetes and also to other long-term conditions. Third, we developed and validated the new severity tool comparing three approaches in large well-defined study cohorts of people with type 2 diabetes. Fourth, we triangulated expert opinion, evidence from literature and database analysis, which resulted in us including more type 2 diabetes-related severity domains compared with previous studies. Fifth, we found an added predictive value of our severity score versus $\mathrm{HbA}_{1 \mathrm{c}}$, which is the mainstay of current clinical decision-making around type 2 diabetes management and monitoring.

There are some limitations to our study. First, we may have missed other severity indicators not recorded in the primary care data. However, using routinely coded data would facilitate automation of disease severity assessment and make our model widely applicable. Second, one of the drawbacks of using comorbidities for assessing severity is their broad definition, which may include conditions not related to diabetes-we used multidisciplinary expert knowledge to maximize the relevance of clinical concepts and codes of the domains included. Third, some of the severity indicators are related to increasing age and deprivation (and possible other covariates), and therefore adjusting for these covariates may have attenuated the risk of outcomes associated with disease severity. Fourth, the lower discrimination observed with the clustered outcome is likely due to an artifact of the way secondary care data are recorded, maybe resulting in the cause of diabetes hospitalization not being added to the record or not added as the main cause for that admission. Fifth, our method might be limited being it health system-specific and more relevant to countries with automated and comprehensive EHRs. However, DISSCO has potential generalizability in non-UK countries as it includes coded clinical data widely found in EHRs adopted for primary care mainly for people with type 2 diabetes, including in countries lacking established EHRs. Finally, external validation of DISSCO in an independent population and a clinical decision support system is needed before reporting its full clinical utility and implications. This can be addressed in a future study.

\section{CONCLUSION}

The prevalence of type 2 diabetes has increased steeply worldwide. This study provides a new type 2 diabetes severity score, DISSCO, which considerably improves the accuracy of predicting hospitalization and death using EHRs routinely collected on type 2 diabetes in primary care. DISSCO has higher predictive value than $\mathrm{HbA}_{1 \mathrm{c}}$ levels on all examined clinical outcomes. More 
generally, we show an overall similar predictive value of incorporating hierarchies of comorbidity severity in models or counts of complications. Disease-specific severity measures using EHRs are feasible, and this is directly relevant to clinical practice and risk stratification, indicating the applicability of big data in the direction of precision medicine. Such disease-specific severity measures can serve as an actionable tool for providing therapeutic targets, have applications in medical research, and have wider implications on individual patient and population healthcare.

\section{Author affiliations}

${ }^{1}$ NIHR School for Primary Care Research, Centre for Primary Care and Health Services Research, Manchester Academic Health Science Centre (MAHSC), The University of Manchester, Manchester, UK

${ }^{2}$ Division of Population Health, Health Services Research and Primary Care, School of Health Sciences, Faculty of Biology, Medicine and Health, Manchester Academic Health Science Centre (MAHSC), The University of Manchester, Manchester, UK ${ }^{3}$ Keele Cardiovascular Research Group, Centre for Prognosis Research, School of Primary, Community and Social Care, Keele University, Stoke-on-Trent, UK ${ }^{4}$ Division of Pharmacy and Optometry, School of Health Sciences, Faculty of Biology, Medicine and Health, Manchester Academic Health Science Centre (MAHSC), The University of Manchester, Manchester, UK

${ }^{5}$ NIHR Greater Manchester Patient Safety Translational Research Centre, The University of Manchester, Manchester, UK

${ }^{6} \mathrm{NIHR}$ Manchester Biomedical Research Centre, Manchester Academic Health Science Centre (MAHSC), Manchester, UK

${ }^{7}$ Centre for Academic Primary Care, Population Health Sciences, Bristol Medical School, University of Bristol, Bristol, UK

${ }^{8}$ School of Primary, Community and Social Care, Faculty of Medicine and Health Sciences, Keele University, Staffordshire, UK

${ }^{9}$ Centre for Biostatistics, School of Health Sciences, Faculty of Biology, Medicine and Health, Manchester Academic Health Science Centre (MAHSC), The University of Manchester, Manchester, UK

${ }^{10}$ Department of Primary Care and Public Health, Brighton and Sussex Medical School, University of Sussex, Falmer, UK

${ }^{11}$ Primary Care Stratified Medicine (PRISM) Research Group, Division of Primary Care, School of Medicine, University of Nottingham, Nottingham, UK

${ }^{12}$ Nuffield Department of Primary Care Health Sciences, University of Oxford, Oxford, UK

${ }^{13}$ Institute of Population Health, University of Liverpool, Liverpool, UK

${ }^{14}$ Division of Informatics, Imaging and Data Sciences, School of Health Sciences, Faculty of Biology, Medicine and Health, Manchester Academic Health Science Centre (MAHSC), The University of Manchester, Manchester, UK

${ }^{15}$ Manchester Diabetes Centre, Manchester University NHS Foundation Trust, Manchester Academic Health Science Centre (MAHSC), Manchester, UK

${ }^{16}$ Division of Diabetes, Endocrinology and Gastroenterology, School of Medical Sciences, Faculty of Biology, Medicine and Health, Manchester Academic Health Science Centre (MAHSC), The University of Manchester, Manchester, UK

Presented at

Some of the findings were presented at the National Institute for Health Research School for Primary Care Research (NIHR SPCR) Showcase, November 2018, London, UK; the Diabetes UK Conference, March 2018, London, UK; and the 55th Annual Meeting for the European Association for the Study of Diabetes (EASD), September 2019, Barcelona, Spain.

Acknowledgements This study is based in part on data from the Clinical Practice Research Datalink obtained under license from the UK Medicines and Healthcare products Regulatory Agency. The data are provided by patients and collected by the NHS as part of their care and support. Hospital Episode Statistics (HES) and Office for National Statistics (ONS) data (copyright (2018)) are reused with permission from the Health and Social Care Information Centre. All rights reserved. The OPCS Classification of Interventions and Procedures, codes, terms and text is Crown copyright (2016) published by the Health and Social Care Information Centre, also known as NHS Digital, and licensed under the Open Government Licence available at www.nationalarchives.gov.uk/doc/open-government-licence/ open-government-licence.htm. The interpretation and conclusions contained in this study are those of the authors alone. The authors thank the participants of the patient and public involvement and engagement (PPIE) event for their time and important feedback, and the two patient partners recruited to codevelop the PPIE event, Mrs Angela Ruddock and Mr Manoj Mistry, which involved codesigning the content for the event and cofacilitating the event on the day.

Contributors EK, MAM, and SSZ designed the study. SSZ extracted and analyzed the data and drafted the manuscript. EK and MAM critically revised the initial versions. DMA, CS, CDM, CAC-G, DR, HVM, NQ, SW, TH, IB, NP, SG, and MKR contributed to interpretation of data and revised the paper for important intellectual content. All authors agreed on the final version of the paper before submission. SSZ is the guarantor. The corresponding author attests that all listed authors meet the authorship criteria and that no others meeting the criteria have been omitted.

Funding This study is funded by the NIHR SPCR (grant number 331). This report is an independent research by the NIHR. The views expressed in this publication are those of the authors and not necessarily those of the NHS, the NIHR or the Department of Health and Social Care. The funder of the study had no role in study design, data collection, data analysis, data interpretation, or writing of the report. The lead author had full access to the data in the study and had final responsibility for the decision to submit for publication. DMA is funded by the NIHR Greater Manchester Patient Safety Translational Research Centre, the NIHR School for Primary Care Research, and the NIHR Manchester Biomedical Research Centre. CDM is funded by the NIHR Collaboration for Leadership in Applied Health Research and Care West Midlands, the NIHR School for Primary Care Research and the NIHR Research Professorship in General Practice (NIHR-RP-2014-04-026). CAC-G is part funded by NIHR Applied Research Collaboration (ARC) West Midlands. NP's time was partially funded by the NIHR Manchester Biomedical Research Centre.

Competing interests DMA reports research grants from Abbvie, Almirall, Celgene, Eli Lilly, Novartis, UCB and the Leo Foundation. CS reports grants from NIHR SPCR, during the conduct of the study and is partially supported by NHS CLAHRC West. CDM is funded by a NIHR Research ship (NIHR-RP- 2014-04026), the NIHR Collaborations for Leadership in Applied Health Research and Care West Midlands and the NIHR School for Primary Care Research. NQ reports grants from the NIHR SPCR and NIHR HTA, during the conduct of the study. SW reports honorarium and independent scientific donation from Amgen and serves as a member of the Clinical Practice Research Datalink Independent Scientific Committee (ISAC) at the UK Medicines and Health Regulatory Agency. IB reports indirect competing interests as Chief Data Scientist Advisor to AstraZeneca via University of Liverpool; NIHR Senior Investigator grant. MKR has received educational grant support from MSD and Novo Nordisk; has modest stock ownership in GSK; and has consulted for Roche.0ther co-authors declare: no support from any organisation for the submitted work; no financial relationships with any organisations that might have an interest in the submitted work in the previous three years; no other relationships or activities that could appear to have influenced the submitted work.

Patient consent for publication Not required.

Ethics approval The study was approved by the Independent Scientific Advisory Committee (ISAC) for MHRA Database Research (protocol number: 17 168). Generic ethical approval for observational research using CPRD with approval from ISAC has been granted by Health Research Authority (HRA) Research Ethics Committee (East Midlands—Derby, REC reference number 05/MRE04/87).

Provenance and peer review Not commissioned; externally peer reviewed.

Data availability statement Data may be obtained from a third party and are not publicly available. All data relevant to the study are included in the article or uploaded as supplementary information. Electronic health records are, by definition, considered sensitive data in the UK by the Data Protection Act and cannot be shared via public deposition because of information governance restriction in place to protect patient confidentiality. Access to data is available only once approval has been obtained through the individual constituent entities controlling access to the data. The primary care data can be requested via application to the Clinical Practice Research Datalink, secondary care data can be requested via application to the Hospital Episode Statistics from the UK Health and Social Care Information Centre, and mortality data are available by application to the UK Office for National Statistics.

Open access This is an open access article distributed in accordance with the Creative Commons Attribution 4.0 Unported (CC BY 4.0) license, which permits others to copy, redistribute, remix, transform and build upon this work for any purpose, provided the original work is properly cited, a link to the licence is given, 
and indication of whether changes were made. See: https://creativecommons.org/ licenses/by/4.0/.

ORCID iD

Salwa S Zghebi http://orcid.org/0000-0002-7978-1094

\section{REFERENCES}

1 World Health Organization (WHO) media centre. Diabetes. Secondary Diabetes [Fact sheet], 2018. Available: http://www.who. int/news-room/fact-sheets/detail/diabetes

2 Hex N, Bartlett C, Wright D, et al. Estimating the current and future costs of type 1 and type 2 diabetes in the UK, including direct health costs and indirect societal and productivity costs. Diabet Med 2012;29:855-62.

3 Walker BR, Colledge NR, Ralston SH, et al. Davidson's principles and practice of medicine. 22nd edn. China: Elsevier, 2014.

4 American Diabetes Association (ADA). Standards of Medical Care in Diabetes - 2018. Diabetes Care 2018;41.

5 American Diabetes Association (ADA). Standards of Medical Care in Diabetes - 2015. Diabetes Care 2015;38:S1-49.

6 Zghebi SS, Steinke DT, Carr MJ, et al. Examining trends in type 2 diabetes incidence, prevalence and mortality in the UK between 2004 and 2014. Diabetes Obes Metab 2017;19:1537-45.

7 Zghebi SS, Rutter MK, Ashcroft DM, et al. Using electronic health records to quantify and stratify the severity of type 2 diabetes in primary care in England: rationale and cohort study design. BMJ Open 2018;8:e020926.

8 Parkerson GR, Broadhead WE, Tse CK. The Duke severity of illness checklist (DUSOI) for measurement of severity and comorbidity. $J$ Clin Epidemiol 1993;46:379-93.

9 Charlson ME, Pompei P, Ales KL, et al. A new method of classifying prognostic comorbidity in longitudinal studies: development and validation. J Chronic Dis 1987;40:373-83.

10 Elixhauser A, Steiner C, Harris DR, et al. Comorbidity measures for use with administrative data. Med Care 1998;36:8-27.

11 Grootenhuis PA, Snoek FJ, Heine RJ, et al. Development of a type 2 diabetes symptom checklist: a measure of symptom severity. Diabet Med 1994;11:253-61.

12 Gini R, Schuemie MJ, Mazzaglia G, et al. Automatic identification of type 2 diabetes, hypertension, ischaemic heart disease, heart failure and their levels of severity from Italian general practitioners' electronic medical records: a validation study. BMJ Open 2016;6:e012413.

13 Young BA, Lin E, Von Korff M, et al. Diabetes complications severity index and risk of mortality, hospitalization, and healthcare utilization. Am J Manag Care 2008;14:15-24.

14 Zghebi SS, Panagioti M, Rutter MK, et al. Assessing the severity of type 2 diabetes using clinical data-based measures: a systematic review. Diabet Med 2019;36:688-701.

15 Herrett E, Gallagher AM, Bhaskaran K, et al. Data resource profile: clinical practice research Datalink (CPRD). Int J Epidemiol 2015;44:827-36
16 Kontopantelis E, Stevens RJ, Helms PJ, et al. Spatial distribution of clinical computer systems in primary care in England in 2016 and implications for primary care electronic medical record databases: a cross-sectional population study. BMJ Open 2018;8:e020738.

17 Williams T, van Staa T, Puri S, et al. Recent advances in the utility and use of the general practice research database as an example of a UK primary care data resource. Ther Adv Drug Saf 2012;3:89-99.

18 Medicines \& Healthcare products Regulatory Agency (MHRA). Clinical Practice Research Datalink. Secondary Clinical Practice Research Datalink, 2018. Available: https://www.cprd.com/home/

19 StataCorp. Stata 15 survival analysis reference manual. secondary Stata 15 survival analysis reference manual, 2017. Available: https:// www.stata.com/manuals/st.pdf

20 StataCorp LLC. Stata statistical software. secondary Stata statistical software, 2018. Available: https://www.stata.com/

21 Benchimol El, Smeeth L, Guttmann A, et al. The reporting of studies conducted using observational Routinely-collected health data (record) statement. PLoS Med 2015;12:e1001885.

22 Gatlin PK, Insel KC. Severity of type 2 diabetes, cognitive function, and self-care. Biol Res Nurs 2015;17:540-8.

23 Wang Q, Wang J, Lin H, et al. Relationship between fat mass and obesity-associated gene expression and type 2 diabetes mellitus severity. Exp Ther Med 2018;15:2917-21.

24 Murrells T, Ball J, Cookson G, et al. Managing diabetes in primary care: how does the configuration of the workforce affect quality of care?: National Nursing Research Unit, King's College London 2013.

25 Eygen Luk V, Patricia S, Luc F, et al. Priorities for diabetes primary care in Europe. Prim Care Diabetes 2008;2:3-8.

26 Spann SJ, Nutting PA, Galliher JM, et al. Management of type 2 diabetes in the primary care setting: a practice-based research network study. Ann Fam Med 2006;4:23-31.

27 Tai T-Y, Chuang L-M, Tsai S-T, et al. Treatment of type 2 diabetes mellitus in a primary care setting in Taiwan: comparison with secondary/tertiary care. J Formos Med Assoc 2006;105:105-17.

28 Mafauzy M. Diabetes control and complications in private primary healthcare in Malaysia. Med J Malaysia 2005;60:212-7.

29 Centre for metabolic bone diseases UoS. FRAX fracture risk assessment tool. Secondary FRAX Fracture Risk Assessment Tool https://www.sheffield.ac.uk/FRAX/index.aspx

30 Hippisley-Cox J, Coupland C, Brindle P. Development and validation of QRISK3 risk prediction algorithms to estimate future risk of cardiovascular disease: prospective cohort study. BMJ 2017;357:j2099.

31 Swedish Society for Diabetology. The Swedish national diabetes register (NDR) secondary the Swedish national diabetes register, 2020. Available: https://www.ndr.nu/\#/variabler

32 Garies S, Birtwhistle R, Drummond N, et al. Data resource profile: national electronic medical record data from the Canadian primary care sentinel surveillance network (CPCSSN). Int J Epidemiol 2017:46:1091-2.

33 American College of Cardiology Foundation. The diabetes collaborative registry. secondary the diabetes collaborative registry, 2020. Available: https://www.ncdr.com/WebNCDR/Diabetes/ publicpage 